\title{
The $n$th Root of NOT Operators of Quantum Computers
}

\author{
Jeffrey Zheng
}

\begin{abstract}
This chapter proposes a novel approach to resolve the $n$th root of NOT problem for quantum computers using $(-1,0,1)$ permutation matrices. Only logic NOT and exchange operations are required. This result provides a complete solution to design and implement the $n$th root of NOT operators of quantum computers.
\end{abstract}

Keywords Quantum simulator - Quantum computation - Square root of NOT $n$-th root of NOT $\cdot$ Permutation matrix $\cdot$ Quantum logic gate

\section{Introduction}

Feynman [1] first proposed 'universal quantum simulator' towards a true quantum computer. Since then, research and development activities of quantum computation and quantum computers have become the new frontal of next-generation computers for two decades [2,3]. Classical quantum mechanics use complex number vectors in Hilbert space to represent quantum states [4]. Any complex number is composed of two parts: a real part and an imaginary part. The imaginary number $i=\sqrt{-1}$ plays the essential role in the quantum mechanics construction. However, the mystery of the imaginary number causes severe difficulties for its manipulation, imagination and understanding [4-6]. Considering that modern computers are constructed by Boolean logic principles, how traditional logic structure is used to implement $\sqrt{-1}$ has been puzzling and deeply entangled in quantum computing for at least two decades [7-10]. Nothing in the published literature has described a way to implement this untamed operator using traditional logic operations $[2,11,12]$.

This work was supported by the Key Project on Electric Information and Next Generation IT Technology of Yunnan (2018ZI002), NSF of China (61362014), Yunnan Advanced Overseas Scholar Project.

\section{J. Zheng $(\bowtie)$}

Key Laboratory of Quantum Information of Yunnan, Yunnan University, Kunming, China e-mail: conjugatelogic@yahoo.com

J. Zheng (ed.), Variant Construction from Theoretical Foundation to Applications, https://doi.org/10.1007/978-981-13-2282-2_17 


\subsection{The Square Root of NOT Problem}

Following traditional logic, negation corresponds to logic NOT $(\neg)$. Initiated by Feynman [1] and further developed by Deutsch [9, 13], this problem has been represented as $\sqrt{\neg}$ 'the Square Root of NOT' as one of the most difficult issues in quantum computation especially in general quantum gates. They suggested resolving $\neg=\left(\begin{array}{ll}0 & 1 \\ 1 & 0\end{array}\right)$ equation using logic operations for the solution. Maglicki and Wang [11] provided an example of how to resolve the problem this way.

Let $\neg$ operation reverse two quantum spin states $|0\rangle=\left(\begin{array}{l}0 \\ 1\end{array}\right), \quad|1\rangle=\left(\begin{array}{l}1 \\ 0\end{array}\right)$,

$$
\begin{aligned}
& \neg|0\rangle=\left(\begin{array}{ll}
0 & 1 \\
1 & 0
\end{array}\right)\left(\begin{array}{l}
0 \\
1
\end{array}\right)=\left(\begin{array}{l}
1 \\
0
\end{array}\right)=|1\rangle \\
& \neg|1\rangle=\left(\begin{array}{ll}
0 & 1 \\
1 & 0
\end{array}\right)\left(\begin{array}{l}
1 \\
0
\end{array}\right)=\left(\begin{array}{l}
0 \\
1
\end{array}\right)=|0\rangle
\end{aligned}
$$

To apply unitary rotational matrices, $\sqrt{\neg}$ operator can be expressed as

$$
\sqrt{\neg}=\frac{1}{\sqrt{2}}\left(\begin{array}{cc}
\mathrm{e}^{i \pi / 4} & \mathrm{e}^{-i \pi / 4} \\
\mathrm{e}^{-i \pi / 4} & \mathrm{e}^{i \pi / 4}
\end{array}\right)=\frac{1}{2}\left(\begin{array}{cc}
1+i & 1-i \\
1-i & 1+i
\end{array}\right)
$$

In the equations, both $\mathrm{e}^{i \pi}$ and $i$ symbols are involved. From a representative viewpoint, equations are useless because the symbols $i$ and $\sqrt{\neg}$ are both logic equivalent. The equations are in circular definitions.

To explore how to use traditional logic implementing $\sqrt{\neg}$, it is necessary to analyse what has been established at the foundation levels of modern complex number construction.

\subsection{Complex Number in History}

The origin and development of complex number has a long and mysterious history [14-16]. In the nineteenth century, Gauss and Euler [15] made their foundation contributions to formally identifying imaginary parts as the most essential components to resolve solutions from $n$th algebraic equations. After their work, the imaginary number has been gradually accepted by mainstream mathematicians to be one of the most important parts of mathematics [15]. Hamilton established consistent operations on complex number in 1837 [17]. He constructed a complex number $a+b i$ as an ordered number pair $(a, b)$.

For example, let $a+b i$ and $c+d i$ be two complex numbers. Four essential operations: $\{ \pm, \bullet, /\}$ can be expressed as 


$$
\begin{aligned}
& (a, b) \pm(c, d)=(a \pm c, b \pm d) \\
& (a, b) \bullet(c, d)=(a c-b d, a d+b c) \\
& \frac{(a, b)}{(c, d)}=\left(\frac{a c+b d}{c^{2}+d^{2}}, \frac{b c-a d}{c^{2}+d^{2}}\right)
\end{aligned}
$$

Using ordered pair representation, complex number operations are firmly established on real number operations. No further mysterious characteristics of imaginary numbers remain in the equations because all operations are well defined in real number construction.

\section{Solution of the Square Root of NOT Problem}

If we apply an imaginary number to an ordered pair, we have

$$
i:(a, b) \rightarrow(-b, a)
$$

When we do not restrict $\sqrt{\neg}$ solution in $\{0,1\}$ field but extend the field to $\{-1$, $0,1\}$. A permutation matrix can be constructed.

Let

$$
\begin{gathered}
I_{2}=\left(\begin{array}{ll}
1 & 0 \\
0 & 1
\end{array}\right), I_{2}^{+}=\left(\begin{array}{cc}
1 & 0 \\
0 & -1
\end{array}\right), I_{2}^{-}=\left(\begin{array}{cc}
-1 & 0 \\
0 & 1
\end{array}\right), \quad Z_{2}=\left(\begin{array}{cc}
0 & 1 \\
-1 & 0
\end{array}\right), Z_{2}^{\perp}=\left(\begin{array}{cc}
0 & -1 \\
1 & 0
\end{array}\right), \\
Z_{2}:(a, b) \rightarrow(-b, a) \\
(-b, a)=(a, b)\left(\begin{array}{cc}
0 & 1 \\
-1 & 0
\end{array}\right)
\end{gathered}
$$

Because $Z_{2}$ provides the same result as the imaginary number when applied to the pair, it is necessary for us to explore $Z_{2}$ features in details.

Two eigenvalues of $Z_{2}$ can be determined from its determinant.

$$
\begin{aligned}
& \left|\lambda I_{2}-Z_{2}\right|=\left|\begin{array}{cc}
\lambda & -1 \\
1 & \lambda
\end{array}\right|=0 \\
& \lambda^{2}+1=0, \quad \lambda^{2}=-1, \quad \lambda= \pm \sqrt{-1}
\end{aligned}
$$

This corresponds to either $\left(\begin{array}{cc}i & 0 \\ 0 & -i\end{array}\right)$ or $\left(\begin{array}{cc}-i & 0 \\ 0 & i\end{array}\right)$ as the solution. There are two unitary matrices $U_{+}, U_{-}$and two Hermite conjugate matrices $U_{+}^{*}, U_{-}^{*}$ undertaken similarity transformation on $Z_{2}$ to produce the two diagonal matrices: 


$$
\begin{aligned}
& i I_{2}^{ \pm}=\left(\begin{array}{cc}
i & 0 \\
0 & -i
\end{array}\right)=U_{+}\left(\begin{array}{cc}
0 & 1 \\
-1 & 0
\end{array}\right) U_{+}^{*} ; \\
& i I_{2}^{\mp}=\left(\begin{array}{cc}
-i & 0 \\
0 & i
\end{array}\right)=U_{-}\left(\begin{array}{cc}
0 & 1 \\
-1 & 0
\end{array}\right) U_{-}^{*}
\end{aligned}
$$

Although three matrices belong to one matrix group under similarity transformation, five matrices can be distinguished without any direct equality.

$$
i I_{2} \neq i I_{2}^{ \pm} \neq Z_{2} \neq i I_{2}^{\mp} \neq-i I_{2}
$$

To apply the five matrices twice separately, they all equal to $-I_{2}$.

$$
\begin{aligned}
& \left( \pm i I_{2}\right)^{2}=\left(\begin{array}{cc} 
\pm i & 0 \\
0 & \pm i
\end{array}\right)\left(\begin{array}{cc} 
\pm i & 0 \\
0 & \pm i
\end{array}\right)=\left(\begin{array}{cc}
-1 & 0 \\
0 & -1
\end{array}\right)=-I_{2} \\
& \left(i I_{2}^{ \pm}\right)^{2}=\left(i I_{2}^{\mp}\right)^{2}=\left(\begin{array}{cc} 
\pm i & 0 \\
0 & \mp i
\end{array}\right)\left(\begin{array}{cc} 
\pm i & 0 \\
0 & \mp i
\end{array}\right)=\left(\begin{array}{cc}
-1 & 0 \\
0 & -1
\end{array}\right)=-I_{2}
\end{aligned}
$$

and

$$
Z_{2}^{2}=\left(\begin{array}{cc}
0 & 1 \\
-1 & 0
\end{array}\right)\left(\begin{array}{cc}
0 & 1 \\
-1 & 0
\end{array}\right)=\left(\begin{array}{cc}
-1 & 0 \\
0 & -1
\end{array}\right)=-I_{2}
$$

Therefore, the $Z_{2}$ matrix is an equivalent form of the imaginary number under the transformation.

For any ordered pair $(a, b)$,

$$
\begin{aligned}
\left(Z_{2}\right)^{2} & :(a, b) \rightarrow(-a,-b) \\
\left(Z_{2}\right)^{2} & :(a, b) \stackrel{Z_{2}}{\longrightarrow}(-b, a) \stackrel{Z_{2}}{\longrightarrow}(-a,-b) \\
\left(Z_{2}\right)^{2} & =-I_{2} \\
Z_{2} & =\sqrt{-I_{2}}
\end{aligned}
$$

So, $\sqrt{\neg}$ operation can be constructed originally from one-one correspondences from the $Z_{2}$ matrix.

Let $\langle x|$ be a quantum state, $\neg\langle x|=\langle\bar{x}|$. For a non-zero element of $Z_{2}$, two values

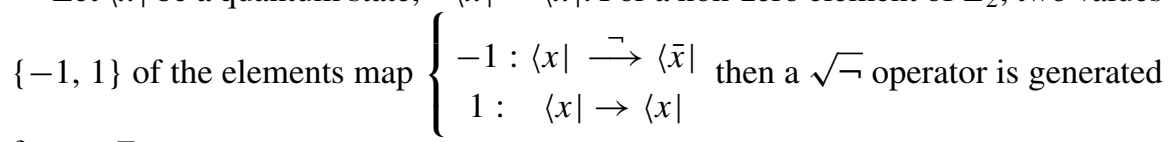
from a $Z_{2}$ operator.

For an ordered state pair $(\langle x|,\langle y|)$, 


$$
(\langle x|,\langle y|) \stackrel{\sqrt{\sqsupset}}{\longrightarrow}(\langle\bar{y}|,\langle x|) \stackrel{\sqrt{\sqsupset}}{\longrightarrow}(\langle\bar{x}|,\langle\bar{y}|)=\neg(\langle x|,\langle y|)
$$

Therefore, $Z_{2}$ is a homologous form of the $\sqrt{\neg}$ operator.

Under this construction, the square root of NOT problem in quantum computation is solved entirely. Only two elementary operations are involved in the transformation: logic NOT operation and pair-state exchange, respectively. They can be implemented readily using traditional logic constructions.

\section{General Solution of the $n$th Root of NOT Operation}

In this part, a general solution of $\sqrt[n]{ }$ 'the $n$th root of NOT' for quantum computers is explored.

Let $J_{n}$ denote a conjugate permutation matrix which contains $n$ columns and $n$ rows and each row (column) has one non-zero element.

$$
J_{n}=\left(J_{i, j}\right), \quad 1=\sum_{i=1}^{n}\left|J_{i, j}\right|=\sum_{i=1}^{n}\left|J_{i, j}\right|, \quad J_{i, j} \in\{-1,0,1\}, i, j \in[1, n]
$$

Let $I_{n}$ be a unit matrix, $I_{i, j}=1, i=j ; I_{i, j}=0, i \neq j, i, j \in[1, n]$.

For example, matrices $\left(\begin{array}{ccc}1 & 0 & 0 \\ 0 & -1 & 0 \\ 0 & 0 & 1\end{array}\right),\left(\begin{array}{ccc}0 & -1 & 0 \\ 1 & 0 & 0 \\ 0 & 0 & 1\end{array}\right),\left(\begin{array}{ccc}0 & 0 & -1 \\ 0 & -1 & 0 \\ 1 & 0 & 0\end{array}\right), I_{3}=\left(\begin{array}{lll}1 & 0 & 0 \\ 0 & 1 & 0 \\ 0 & 0 & 1\end{array}\right)$ are $J_{n}$ matrices.

Let $P_{n}$ be a $(0,1)$-permutation matrix in which each column (row) contains only one element, and $P S(n)$ denote a permutation space containing all $P_{n}$ matrices.

Let $J S(n)$ denote a conjugate permutation space.

Lemma For a given n, PS(n) contains a total number of $n$ ! distinguishable matrices, that is, $|P S(n)|=n !$.

Theorem For a given n, JS(n) contains a total number of $2^{n} n$ ! distinguishable matrices, $|J S(n)|=2^{n} n$ !.

Proof Each non-zero element of $J_{n}$ has two values $\{-1,1\}$, and $n$ different elements have $2^{n}$ selections. The $n$ elements can select a total number of $n$ ! different positions. Both symbol and position selections are independent, and each combination determines a $J_{n}$ matrix. So there are $2^{n} n$ ! distinguishable matrices.

Corollary $J S(n)$ is a matrix space that is $2^{n}$ times larger than $P S(n)$.

Theorem A matrix group of simple rotation in $J S(n)$ may contain $2 n$ distinguishable matrices. 
Proof Using a rotation matrix $Z_{n} \in J S(n)$,

$$
\begin{gathered}
Z_{n}=\left(\begin{array}{ccccccc}
0 & 1 & 0 & 0 & \ldots & 0 & 0 \\
0 & 0 & 1 & 0 & \ldots & 0 & 0 \\
0 & 0 & 0 & 1 & \ldots & 0 & 0 \\
& & & \ldots & \\
\ldots & & & \ldots & \ldots \\
0 & 0 & 0 & 0 & \ldots & 0 & 1 \\
-1 & 0 & 0 & 0 & \ldots & 0 & 0
\end{array}\right), J_{i, i+1}=1, i \in[1, n], J_{n, 1}=-1 \text { and a vector } \\
X=\left(\begin{array}{llll}
1 & 2 & 3
\end{array} \ldots n-1 n\right) .
\end{gathered}
$$

To apply $2 n Z_{n}$ matrices sequentially to the vector $X$, the following $2 n$ vectors are produced:

$$
\left(\begin{array}{c}
X=X Z_{n}^{2 n} \\
X Z_{n} \\
\ldots \\
X Z_{n}^{n} \\
X Z_{n}^{n+1} \\
\ldots \\
X Z_{n}^{2 n-1}
\end{array}\right)=\left(\begin{array}{ccccccc}
1 & 2 & 3 & \ldots & n-2 & n-1 & n \\
-n & 1 & 2 & \ldots & n-3 & n-2 & n-1 \\
& \ldots & \ldots & & \ldots & \\
-1 & -2 & -3 & \ldots & -n+2 & -n+1 & -n \\
n & -1 & -2 & \ldots & -n+3 & -n+2 & -n+1 \\
& \ldots & & \ldots & & \ldots & \\
2 & 3 & 4 & \ldots & n-1 & n & -1
\end{array}\right)
$$

That is, 2n distinguishable matrices $\left\{Z_{n}^{j}\right\}_{j=1}^{2 n}, Z_{n}^{0}=Z_{n}^{2 n}=I_{n}$ are included.

Because of $X \stackrel{Z_{n}^{n}}{\longrightarrow}-X \stackrel{Z_{n}^{n}}{\longrightarrow} X$, there are $Z_{n}^{n}=-I_{n}$ and $Z_{n}^{2 n}=I_{n}$, that is, $Z_{n}^{n}=-I_{n}$.

Theorem For a $Z_{n}$, there are $n$ eigenvalues $\left\{\lambda_{i}\right\}_{i=1}^{n}, \lambda_{i}=\sqrt[n]{-1}, i \in[1, n]$.

Proof

$$
\left|\lambda I_{n}-Z_{n}\right|=\left|\begin{array}{cccccc}
\lambda & -1 & 0 & \ldots & 0 & 0 \\
0 & \lambda & -1 & \ldots & 0 & 0 \\
\ldots & & \ldots & \ldots \\
0 & 0 & 0 & \ldots & \lambda & -1 \\
1 & 0 & 0 & \ldots & 0 & \lambda
\end{array}\right|=\lambda^{n}+1=0
$$

Therefore, $Z_{n}=\sqrt[n]{-I_{n}}$.

For non-zero values, $\left\{\begin{array}{c}1:\langle x| \rightarrow\langle x| \\ -1:\langle x| \rightarrow\langle\bar{x}|\end{array}\right.$ map $Z_{n} \rightarrow \sqrt[n]{ }$. 
Theorem For any state vector $X, X(\sqrt[n]{\neg})^{n}=\neg X$.

Proof

$$
\left(\begin{array}{c}
X \\
X \sqrt[n]{\neg} \\
X \sqrt[n]{\neg}^{n-1} \\
X \sqrt[n]{\neg}^{n}=\neg X
\end{array}\right)=\left(\begin{array}{ccc}
\langle 1|\langle 2|\langle 3| \ldots & \langle n| \\
\langle\bar{n}|\langle 1|\langle 2| \ldots & \langle n-1| \\
\ldots \ldots & \ldots \ldots & \ldots \\
\langle\overline{2}|\langle\overline{3}|\langle\overline{4}| \ldots & \langle 1| \\
\langle\overline{1}|\langle\overline{2}|\langle\overline{3}| \ldots & \langle\bar{n}|
\end{array}\right) .
$$

\section{Conclusion}

Using $(-1,0,1)$ permutation matrices as basic tools, the $n$th root of NOT operators for quantum computers can be constructed and implemented by the traditional logic structure. Considering that this problem has puzzled advanced research of quantum computer for 20 years, this solution can provide quantum computer designers to practically implement quantum computers using traditional logic. The details of this construction will investigate in other places and the relationships among conjugate logic, quantum logic, quantum gates and complex number structures will be explored for foundation of Quantum computers and quantum computation of future computers.

Acknowledgements Thanks to Dr. G. Liu, Mrs. W. Macmillan, Dr. C. Liu, Dr. A. Tharumarajah and Dr. S. Yang for their invaluable comments, suggestions and careful corrections. Supported, in part by CRC for Intelligent Manufacturing Systems and Technologies.

\section{References}

1. R.P. Feynman, Simulating physics with computers. Int. J. Theor. Phys. 21(6/7), 467-488 (1982)

2. M.A. Nielsen, I.L. Chuang, Quantum Computation and Quantum Information (Cambridge University Press, 2000)

3. J. Preskill, S.P. Trivedi, M.B. Wise, Phys. Lett. B 223, 26 (1989)

4. R.P. Feynman, R.B. Leighton, M.S. Sands, The Feynman Lectures on Physics, vol. 3 (AddisonWesley, 1989)

5. C. Bennett, Logic reversibility of computation. IBM J. Res. Dev. 17, 525-532 (1973)

6. D. Bouwmeester, A. Ekert, A. Zeilinger, The Physics of Quantum Information (Springer, 2000)

7. A. Barenco, C.H. Bennett, R. Cleve, D.P. DiVincenzo, N. Margolus, P. Shor, T. Sleator, J.A. Smolin, H. Weinfurter, Elementary gates for quantum computation. Phys. Rev. A 52(5), 3457-3467 (1995) 
8. D.W. Leung, I.L. Chuang, F. Yamaguchi, Y. Yamamoto, Efficient implementation of coupled logic gates for quantum computation. Phys. Rev. A 61 (2000)

9. D. Deutsch, Quantum theory, the Church-Turing principle and the universal quantum computer. Proc. R. Soc. Lond. A 400, 97-117 (1985). http://doi.org/10.1098/rspa.1985.0070. Published 8 July 1985

10. A.C.C. Yao, Quantum circuit complexity, in Proceedings of the 34th IEEE Symposium on Foundations of Computer Science (IEEE Computer Society Press, 1993), pp. 352-360

11. Z. Meglicki, Z. Wang, Quantum Computing and Topological Quantum Computing (2001)

12. C.P. Williams, S.H. Clearwater, Explorations in Quantum Computing (Springer, Berlin, 1998)

13. D. Deutsch, R. Jozsa, Rapid solution of problems be quantum computation. Proc. R. Soc. Lond. 439A, 553-558 (1992)

14. F.J. Budden, Complex Numbers and their Applications (Longmans Green and Co Ltd. 1968)

15. M. Kline, Mathematical Thought From Ancient to Modern Times, (Oxford University Press, 1972)

16. J.M. Olmsted, Calculus with Analytic Geometry, vol. II (Meredith Publishing Company, 1966)

17. W.R. Hamilton, Theory of conjugate functions or algebraic couples; with a preliminary essay on algebra as the science of pure time. Trans. Royal Irish Academy Vol. XVII, 293-422. (The Mathematical Papers of Sir William Rowan Hamilton, Vol. III Algebra, edited for Royal Irish Academy, 3-100) (1837)

18. J. Preskill, A. Kiteav, Quantum Information and Computation, Lecture Notes for Physics 229, http://www.theory.caltech.edu/people/preskill/ph229

Open Access This chapter is licensed under the terms of the Creative Commons Attribution 4.0 International License (http://creativecommons.org/licenses/by/4.0/), which permits use, sharing, adaptation, distribution and reproduction in any medium or format, as long as you give appropriate credit to the original author(s) and the source, provide a link to the Creative Commons license and indicate if changes were made.

The images or other third party material in this chapter are included in the chapter's Creative Commons license, unless indicated otherwise in a credit line to the material. If material is not included in the chapter's Creative Commons license and your intended use is not permitted by statutory regulation or exceeds the permitted use, you will need to obtain permission directly from the copyright holder.

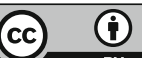

